\title{
Cathode ion bombardment in rf photoguns
}

\author{
E. Pozdeyev, ${ }^{*}$ D. Kayran, and V. N. Litvinenko \\ Brookhaven National Laboratory, Upton, New York 11973-5000, USA
}

(Received 9 September 2008; published 29 April 2009)

\begin{abstract}
We use the ponderomotive potential of the rf field to accurately describe the motion of ions in an rf gun. We apply the method to the BNL 1/2-cell superconducting radio-frequency photogun and demonstrate that a significant portion of ions produced in the gun can reach the cathode if no special precautions are taken. Also, we propose simple mitigation techniques that can reduce the rate of ion bombardment.
\end{abstract}

DOI: 10.1103/PhysRevSTAB.12.043501

PACS numbers: 29.25.Bx, 85.60.Ha

\section{INTRODUCTION}

eRHIC is a future electron-ion collider designed at Brookhaven National Laboratory (BNL) [1]. The current eRHIC design is based on a superconducting energy recovering linac (ERL). To achieve a projected luminosity of $10^{33} \mathrm{~cm}^{-2} \mathrm{sec}^{-1}$, eRHIC requires a polarized electron beam with a current of 50 to $250 \mathrm{~mA}$ and a bunch charge of 5 to $20 \mathrm{nC}$. A superconducting radio-frequency (SRF) photogun with a negative electron affinity GaAs cathode can be a viable option for the eRHIC electron source. An SRF gun has several significant advantages when compared to a normal-conducting rf gun. First, applying superconductivity solves the problem of power losses without sacrificing the accelerating gradient. Second, SRF technology naturally provides the ultrahigh vacuum environment required for the prolonged lifetime of the photocathode. An effort to explore feasibility of using a GaAs cathode in an SRF gun has been undertaken at BNL [2]. Details of earlier experiments with normal-conducting guns can be found in $[3,4]$.

GaAs cathodes are used extensively in static voltage (DC) photoinjectors, polarized and nonpolarized. Operational experience with DC guns has demonstrated that ion backbombardment is the major cause of degradation of the quantum efficiency (QE) of GaAs cathodes. Numerical simulations described in publications [4,5] revealed that ion backbombardment also can occur in $\mathrm{rf}$ guns, possibly limiting the lifetime of cathodes. Although numerical simulations are useful for a specific gun, they are hard to extrapolate to other guns if the scaling laws are not known.

In this paper, we apply the method of a rapidly oscillating field to analyze motion of ions in an rf gun. Kapitza originally proposed this method in $1951[6,7]$ to treat motion of a pendulum with a periodically moving suspension point. He based his analysis on calculating the time average of a force over a period of fast oscillations. Boot and Harvie [8] and Gaponov and Miller [9] applied the same approach to charged particles in oscillating electro-

\footnotetext{
*pozdeyev@bnl.gov
}

magnetic fields. We briefly describe the method in Sec. II, emphasizing the importance of initial conditions in Sec. II B. In Sec. III, we examine the ion motion in the BNL 1/2-cell SRF gun and, in Sec. IV, discuss possible mitigation techniques. We treat the motion of ions in the presence of an external magnetic field in Appendix D.

We would like to note that electron backbombardment and multipacting also can affect the cathode lifetime in an rf gun. However, we do not discuss this phenomenon herein.

\section{MOTION OF IONS IN A RAPIDLY OSCILLATING RF FIELD}

\section{A. Effective potential energy of an ion in an rf field}

An accurate analytical solution of the equation of ion motion in an rf gun is impossible in the general case. However, this equation can be solved iteratively using the method of a rapidly oscillating field. Accordingly, the radius vector of an ion can be expressed as a sum of a fast oscillating term, a, and a term describing the ion motion averaged over the fast oscillations, $\mathbf{x}(t)=\overline{\mathbf{r}(t)}: \mathbf{r}=\mathbf{x}+\mathbf{a}$. If the rf electric field has the following form, $\mathbf{E}=\mathcal{E}(\mathbf{r}) \times$ $\cos (\omega t+\psi)$, the fast oscillating term is given by

$$
\mathbf{a}=-\hbar^{2} \frac{q \mathcal{E} \cos (\omega t+\psi)}{m c^{2}}
$$

and the equation for the averaged motion is given by

$$
\ddot{\mathbf{x}}=-\frac{\lambda^{2} c^{2}}{4}\left(\frac{q}{m c^{2}}\right)^{2} \nabla \mathcal{E}^{2},
$$

where $m$ and $q$ are the mass and charge of the ion, respectively, $c$ is the speed of light, and $\lambda$ is the rf wavelength divided by $2 \pi$. The method is applicable provided that the amplitude of the fast oscillations is small compared to the characteristic size of the inhomogeneity of the rf field, $L$ : $|\mathbf{a}| / L \ll 1$.

Equation (2) shows that the effect of the rf field averaged over the fast oscillations can be described by the effective potential energy, 


$$
U_{e}=\frac{m c^{2}}{4}\left(\frac{\lambda q \mathcal{E}}{m c^{2}}\right)^{2}=\frac{Z^{2}}{A} \frac{m_{u} c^{2}}{4}\left(\frac{\lambda e \mathcal{E}}{m_{u} c^{2}}\right)^{2},
$$

where $Z$ and $A$ are the ion charge and mass states, respectively, $e$ is the elementary charge, and $m_{u} c^{2}$ is the energy equivalent of the atomic mass unit, $931.5 \mathrm{MeV}$. The potential energy (3) frequently is referred to as the "ponderomotive" potential energy.

In the presence of an external electromagnetic field, the general form of the Lagrange function of the averaged motion is given by

$$
L=T_{e}-U_{e}-q \Phi+\frac{q}{c} \mathbf{A} \cdot \dot{\mathbf{x}},
$$

where we introduced the effective kinetic energy $T_{e}=$ $m \dot{\mathbf{x}} / 2$ and expressed the scalar and vector potentials of the external field as $\Phi$ and $\mathbf{A}$, respectively. Provided that the amplitude of the electric field does not depend on time, the total effective energy is an integral of motion:

$$
T_{e}+U_{e}+q \Phi=\text { const. }
$$

\section{B. Initial conditions and effective kinetic energy of ions}

In treating the ion motion, we assume that the ions are generated only in collisions of the electron beam with the residual gas. The initial velocity of an ion after ionization is given by

$$
\dot{\mathbf{r}}_{0}=\dot{\mathbf{x}}_{0}+\dot{\mathbf{a}}_{0}=\dot{\mathbf{x}}_{0}+\lambda c \frac{q \mathcal{E} \sin \left(\phi_{0}\right)}{m c^{2}},
$$

where $\phi_{0}$ is the rf phase at which ionization happens. The ion gains its initial velocity $\dot{\mathbf{r}}_{0}$ interacting with the collective electric field of the bunch that creates the ion. We consider this process in detail in Appendix A. Until then, we assume that intensity of electron bunches is sufficiently low and neglect their field. Additionally, the ion can obtain energy during the ionization process. The energy transferred in the ionization process is mostly absorbed by knocked out electrons [10]. Thus, the cross section of collisions with a large energy transfer to an ion is much smaller than the ionization cross section. Therefore, the energy transfer in the ionization process can be neglected. Assuming $\dot{\mathbf{r}}_{0}=0$ exactly, we find $\dot{\mathbf{x}}_{0}$ :

$$
\dot{\mathbf{x}}_{0}=-\lambda c \frac{q \mathcal{E} \sin \left(\phi_{0}\right)}{m c^{2}} .
$$

The associated effective kinetic energy is given by

$$
T_{e 0}=m \frac{\dot{\mathbf{x}}_{0}^{2}}{2}=\frac{m c^{2}}{2}\left(\frac{\lambda q \mathcal{E}}{m c^{2}}\right)^{2} \sin ^{2}\left(\phi_{0}\right)=2 U_{e} \sin ^{2}\left(\phi_{0}\right) .
$$

We note that this analysis can be applied without any modification to ions produced in collisions with multipactor and dark current electrons. However, in this paper, we assume that high levels of multipacting and dark current are not acceptable for a continuous-wave (CW) SRF photo- gun with a GaAs cathode because of the strict requirements on vacuum level, beam losses, and power losses in the cavity. Therefore, we neglect ionization from mutlipacting and the dark current.

\section{BNL 1/2-CELL SRF GUN}

Brookhaven National Laboratory and Advanced Energy Systems, Inc. are jointly developing a 1/2-cell superconducting radio-frequency (SRF) photogun [11]. The gun will serve as an injector for the BNL R\&D ERL [12] and a test bed for different types of photocathodes. Figure 1 shows the SUPERFISH [13] model of the gun. Table I lists main gun parameters with their nominal values. Figure 2 shows the accelerating electric field on the gun axis calculated by SUPERFISH.

The operational residual gas pressure in the gun is expected to be about $10^{-11}$ Torr or lower. At this pressure, the residual gas mostly will consist of hydrogen. Using (1) and the gun parameters listed in Table I, we can estimate that the amplitude of oscillations of $\mathrm{H}_{2}^{+}$ions in the gun is less than $60 \mu \mathrm{m}$, i.e., well below the characteristic spatial

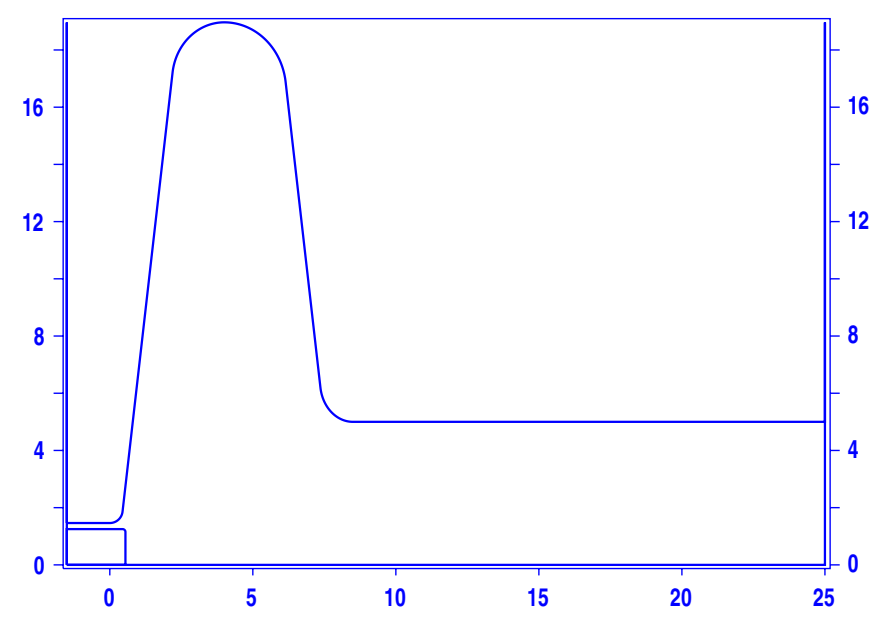

FIG. 1. (Color) SUPERFISH model of the BNL 1/2-cell SRF gun. The horizontal axis corresponds to the gun axis. The vertical axis is the radius. All dimensions are in centimeters. The cathode is at the lower left corner of the figure.

TABLE I. Parameters of the BNL 1/2-cell SRF gun with nominal values. These numbers are used in the paper. The numbers in parentheses show values after a possible laser upgrade.

\begin{tabular}{lc}
\hline \hline Parameter & Value \\
\hline Beam energy $(\mathrm{MeV})$ & 2.0 \\
$\mathcal{E}_{\text {max }}(\mathrm{MeV} / \mathrm{m})$ & 29 \\
$F_{\text {rf }}(\mathrm{MHz})$ & 703.75 \\
$F_{\text {bunch }}(\mathrm{MHz})$ & $9.38(352)$ \\
$q_{\text {bunch }}(\mathrm{nC})$ & $0.7-5$ \\
$I_{\text {beam }}(\mathrm{mA})$ & $7-50(500)$ \\
\hline \hline
\end{tabular}




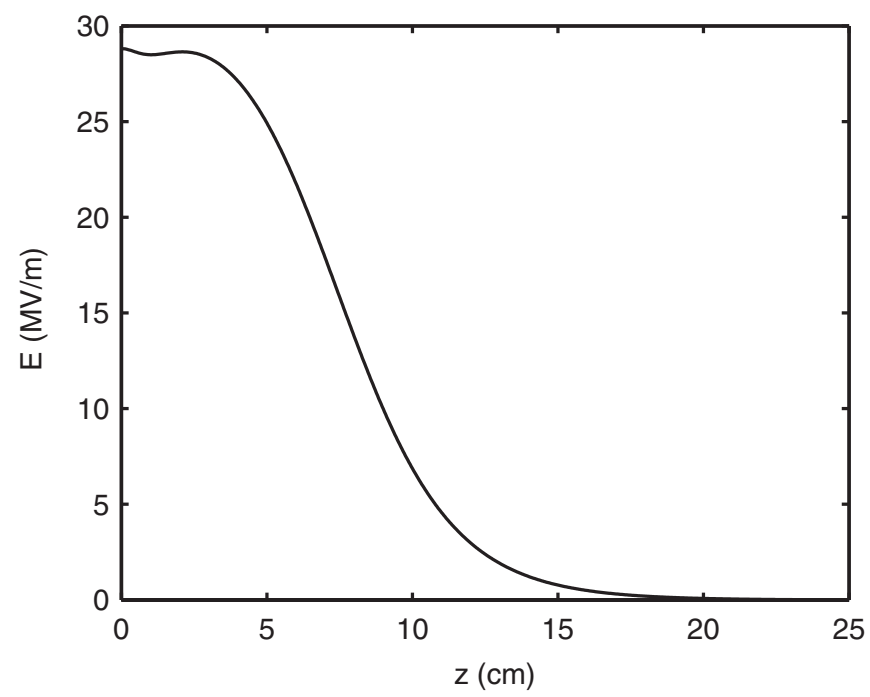

FIG. 2. Longitudinal electric field on the gun axis. The longitudinal coordinate $z=0$ corresponds to the cathode surface.

scale of field inhomogeneity. Thus, we can apply the method of a rapid oscillating field to the BNL gun.

\section{A. Motion of ions on gun axis}

The axial symmetry of the gun requires that the rf electric field has only the $z$ component and the rf magnetic field is zero on the gun axis. Thus, the ion motion on the gun axis is one dimensional. The electric field calculated by SUPERFISH (Fig. 2) and Eq. (3) yields the effective potential energy. The program PARMELA [14] was used to calculate the rf phase at which electron bunches pass a given coordinate. Under the assumption that bunches were short, this simulation yielded the ionization phase $\phi_{0}$ as a function of $z$ shown in Fig. 3. In this simulation, the initial beam phase was chosen to minimize the beam emittance.

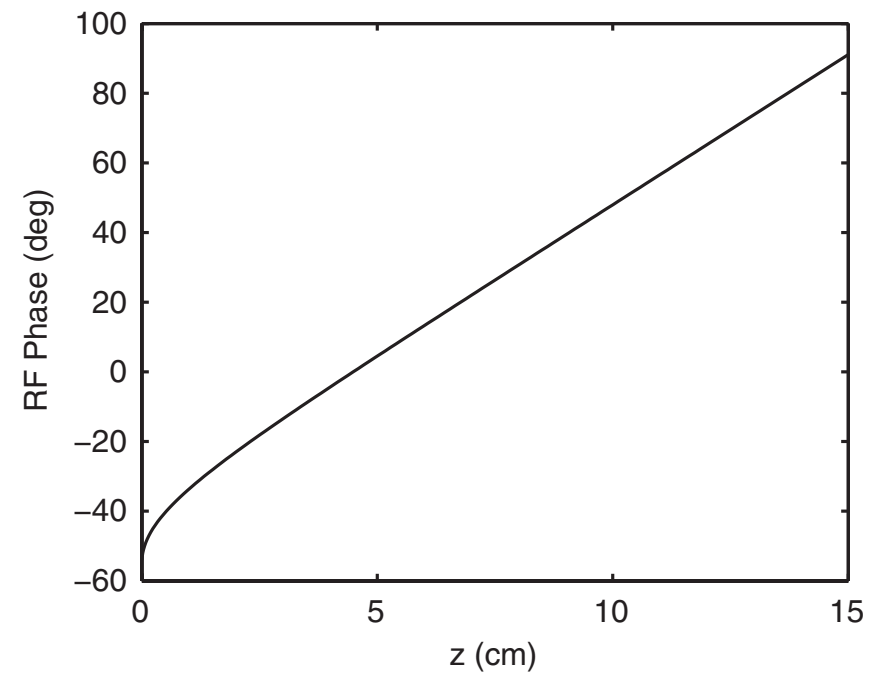

FIG. 3. The rf phase at which electron bunches pass a given coordinate $z$.

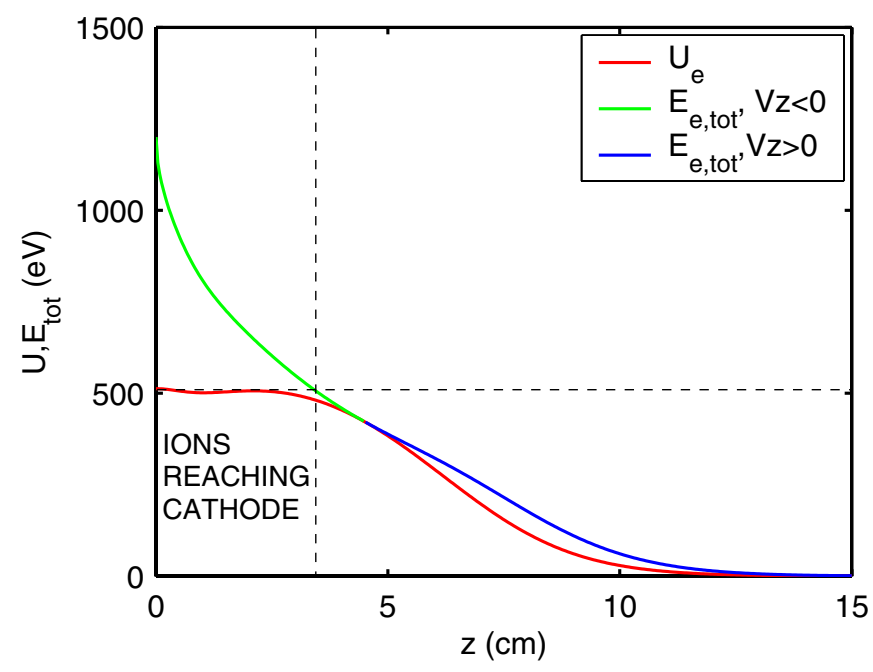

FIG. 4. (Color) The effective potential energy (red curve) and the total effective energy (green and blue curves) of $\mathrm{H}_{2}^{+}$ions in the BNL 1/2-cell SRF gun. The green and blue curves, respectively, show the total energy of ions with the effective velocity $\dot{\mathbf{x}}_{0}$ pointing towards the cathode and away from the cathode. Ions with an initial velocity directed towards the cathode and whose total energy is higher than the maximum potential energy will reach the cathode.

Equation (8) and $\phi_{0}(z)$ yield the initial effective kinetic energy $T_{e 0}$ as a function of the ionization coordinate.

Figure 4 shows the effective potential energy (in red) of $\mathrm{H}_{2}^{+}$ions on the gun axis. Also, Fig. 4 shows the total effective energy of $\mathrm{H}_{2}^{+}$ions as a function of the ionization coordinate. The total energy curve is divided into two branches shown by different colors: Ions whose velocity $\dot{\mathbf{x}}_{0}$ points towards the cathode belong to the green branch $(z<4.5 \mathrm{~cm})$ while those whose velocity points out of the gun belong to the blue branch $(z>4.5 \mathrm{~cm})$. At $z \approx$ $4.5 \mathrm{~cm}$, the total effective energy is equal to the potential energy that corresponds to $\dot{\mathbf{x}}_{0}=0$.

The total effective energy of ions originating at $z<$ $3.4 \mathrm{~cm}$ is larger than the effective potential energy at the cathode. Also, their velocity $\dot{\mathbf{x}}_{0}$ is directed towards the cathode. Thus, those ions originating at $z<3.4 \mathrm{~cm}$ will reach the cathode. The effective kinetic energy $T_{e}$ of ions bombarding the cathode is less than $700 \mathrm{eV}$. All other ions produced at $z \geq 3.4 \mathrm{~cm}$ will be expelled from the gun.

\section{B. Motion of ions off axis}

Typically, the radial beam size and the cathode are much smaller than the reduced rf wavelength $\lambda$. Therefore, we consider only ion trajectories with a small radial deviation from the gun axis compared to $\lambda$. The electric field off the gun axis can be expressed via the electric field on the gun axis and its derivatives with respect to $z$. Using the notation

$$
\mathcal{E}_{a}(z)=\mathcal{E}_{z}(z, r=0)
$$

we can write the electric field $\mathcal{E}(z, r)$ to the second order in 
$r$ as

$$
\begin{aligned}
& \mathcal{E}_{z}(z, r)=\mathcal{E}_{a}-\frac{1}{4}\left(\mathcal{E}_{a}^{\prime \prime}+\frac{\mathcal{E}_{a}}{\hbar^{2}}\right) r^{2}+\cdots \\
& \mathcal{E}_{r}(z, r)=-\frac{\mathcal{E}_{a}^{\prime}}{2} r+\cdots
\end{aligned}
$$

where ' stands for $d / d z$. Then, $\mathcal{E}^{2}$ can be written off axis as

$$
\begin{aligned}
\mathcal{E}^{2} & =\mathcal{E}_{z}^{2}+\mathcal{E}_{r}^{2}=\left(\mathcal{E}_{a}+\delta \mathcal{E}_{z}\right)^{2}+\mathcal{E}_{r}^{2} \\
& \approx \mathcal{E}_{a}^{2}+2 \mathcal{E}_{a} \delta \mathcal{E}_{z}+\mathcal{E}_{r}^{2} .
\end{aligned}
$$

Equations (10a), (10b), (11), and (3) yield the effective potential energy to the second order in $r$ as

$$
U_{e}=\frac{m c^{2}}{4}\left(\frac{\lambda q}{m c^{2}}\right)^{2}\left[\mathcal{E}_{a}^{2}-\frac{\mathcal{E}_{a}}{2}\left(\mathcal{E}_{a}^{\prime \prime}+\frac{\mathcal{E}_{a}}{\lambda^{2}}\right) r^{2}+\frac{\left(\mathcal{E}_{a}^{\prime}\right)^{2}}{4} r^{2}\right]
$$

The radial motion of an ion averaged over fast oscillations is described by the Lagrange equation,

$$
\frac{d}{d t} \frac{\partial L}{\partial \dot{r}}=\frac{\partial L}{\partial r}
$$

with the Lagrangian $L$ given by (4). Assuming that ions originate at rest, the angular component of the ion velocity and the corresponding angular momentum can be neglected, and the equation for the average ion radius can be written as

$$
m \ddot{r}=-\frac{\partial U_{e}}{\partial r} .
$$

Equation (14) has to be solved simultaneously with the Lagrange equation for $z$. However, because we are interested only in ion trajectories with a small deviation from the gun axis, we can neglect the effect of the radial motion on the axial motion. That is, we can solve (14) assuming that the axial motion does not depend on $r$ and use the value of $\dot{x}_{z}$ on the gun axis.

Generally, Eq. (14) has to be solved numerically. To solve Eq. (14) we wrote a short $\mathrm{C}++$ code using the $1 \mathrm{st}$ order Euler method. Higher order integration methods were unnecessary because the ion trajectories were smooth and the curvature of the trajectories was slight. The blue curve in Fig. 5 shows deviation of ion trajectories from the initial radius calculated at the cathode and normalized to the initial radius vs the ionization coordinate. Accordingly, we see that the radius of the ion trajectories at the cathode does not exceed the initial radius by more than $17 \%$ for almost all ions that originate between 0 and $3.4 \mathrm{~cm}$.

A solution of (14) also can be found by iterations. If the trajectory radius changes little, we can limit the solution to the first iteration and solve the problem analytically. This approach is described in detail in Appendix C. The green curve in Fig. 5 depicts the deviation of ion trajectories at the cathode given by the first iteration [Eq. (C5)]. We employed the mathematical package MATHCAD [15] and

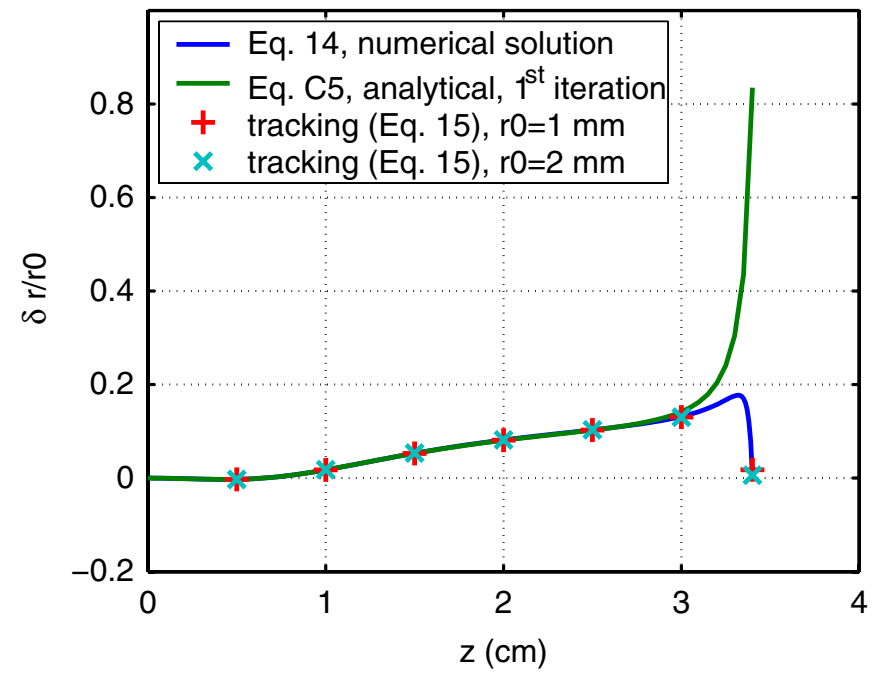

FIG. 5. (Color) Normalized deviation of ion trajectories from the initial radius at the cathode as a function of the ionization coordinate. Tracking results for $r_{0}=1 \mathrm{~mm}$ and $r_{0}=2 \mathrm{~mm}$ practically coincide with each other, confirming the linearity of ion motion close to the gun axis.

the field on the gun axis (Fig. 2) to calculate the integrals in Eq. (C5). This result agrees well up to $z \approx 3 \mathrm{~cm}$ with the numerical solution of (14) described in the previous paragraph. The first iteration predicts a large orbital displacement for $z>3$ and, therefore, cannot be used for ions originating in that region.

This analysis does not include an external magnetic field. This is a valid approach for an SRF gun because the external magnetic field is vanishingly small inside the gun. For a normal-conducting gun, the field of an emittance compensating solenoid can penetrate the rf cavity and affect the ion motion. We consider an external solenoidal magnetic field in Appendix D.

\section{Validation by tracking}

To test the results described above a short $\mathrm{C}++$ tracking code was developed. The code calculated ion trajectories in the two-dimensional rf field maps generated by SUPERFISH. Using the classical 4th order Runge-Kutta integration method [16], the code solved the following system of equations:

$$
\begin{aligned}
\frac{d z}{d \phi} & =\tilde{p}_{z} \\
\frac{d \tilde{p}_{z}}{d \phi} & =\frac{Z e E_{z}}{A m_{u} c^{2}} \lambda^{2} \\
\frac{d r}{d \phi} & =\tilde{p}_{r} \\
\frac{d \tilde{p}_{r}}{d \phi} & =\frac{Z e}{A m_{u} c^{2}}\left(E_{r} \lambda-\tilde{p}_{z} B_{\varphi}\right) \lambda,
\end{aligned}
$$

where $\phi$ is the rf phase used as the independent variable. 
$\tilde{p}_{r, z}$ is related to the particle momentum $p_{r, z}$ as

$$
\tilde{p}_{r, z}=\frac{p_{r, z}}{m \omega},
$$

where $\omega$ is the rf angular frequency. We note that the magnetic field term cannot be neglected in (15d) because its effect on the averaged radial motion of ions is comparable to that of the inhomogeneity of the electric field. Although this seemingly contradicts Eq. (2), it is trivial to show that the term $\nabla \mathcal{E}^{2}$ in (2) includes the magnetic force.

In simulating the beam dynamics in the BNL gun, test particles were launched from nodes of a rectangular $r-z$ mesh. The starting points were uniformly distributed over the interval $z=0.5-10 \mathrm{~cm}$ with steps of $5 \mathrm{~mm}$. Radially, the nodes were located at $r=0,1$, and $2 \mathrm{~mm}$. The simulated axial motion agreed very well with predictions of the 1D model described in Sec. III A: Ions originating between the cathode and $z \approx 3.4 \mathrm{~cm}$ reached the cathode while those originating at $z>3.4 \mathrm{~cm}$ left the gun. The result of the simulation of the radial motion is shown in Fig. 5. As expected, the tracking result is in good agreement with the numerical solution of (14). Also, it agrees well with the first iteration for $z \leq 3 \mathrm{~cm}$. Finally, we point out that the trajectories corresponding to different initial radii normalized to the initial radius yield almost the same result, thereby, proving the linearity of the radial ion motion.

\section{Rate of ion bombardment. Comparison to a DC gun}

The number of ions bombarding the cathode normalized to the extracted charge is given by

$$
\frac{d N}{d Q}=\frac{n_{i}}{e} \int_{0}^{D} \sigma[E(z)] d z
$$

where $n_{i}$ is the particle density of the residual gas, $\sigma$ is the ionization cross section, and $D$ is the distance from which ions can reach the cathode. Figure 6 shows the $\mathrm{H}_{2}$ ionization cross section as a function of the electron beam energy [17]. For the BNL gun, we assumed a residual hydrogen pressure of $5 \times 10^{-12}$ Torr and the distance $D$ of $3.4 \mathrm{~cm}$. The beam energy as a function of $z$ was calculated by the code PARMELA. For these parameters, Eq. (17) yielded

$$
\left(\frac{d N}{d Q}\right)_{\mathrm{BNL} \mathrm{SRF}}=1.7 \times 10^{6} \text { ions } / \mathrm{C} .
$$

According to Secs. III A and III B almost all these ions will strike the cathode.

We can compare this number to the number of ions produced in a high voltage DC gun. For this example, we have chosen the following parameters: Beam energy was $650 \mathrm{keV}$, the accelerating gap was $5 \mathrm{~cm}$, and the residual gas pressure was the same as that in the example of the BNL gun, viz., $5 \times 10^{-12}$ Torr. With these parameters, Eq. (17) yielded

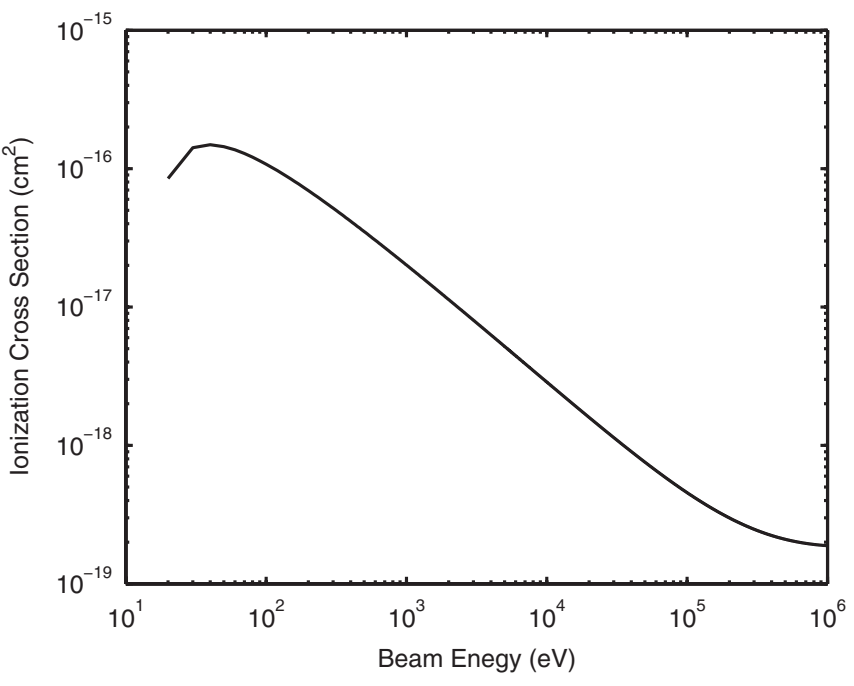

FIG. 6. $\mathrm{H}_{2}$ ionization cross section as a function of the beam energy.

$$
\left(\frac{d N}{d Q}\right)_{\mathrm{HV} \mathrm{DC}}=2.4 \times 10^{6} \text { ions } / \mathrm{C} .
$$

In principle, ions produced in a transfer line can be trapped in the beam and travel towards a gun as described in [18]. However, trapped low energy ions cannot reach the cathode in the BNL gun because of the effective potential barrier produced by the accelerating rf field. In a DC gun, the flux of trapped ions can be eliminated by biasing the anode to a positive potential of a few hundred or thousand volts as proposed in [18].

Expressions (18) and (19) predict similar rates of ion bombardment in the BNL gun and in the DC example. However, it is difficult to accurately compare the cathode lifetime of DC and rf guns based on the overall rate of cathode bombardment. First, the ion energy spectra in the two differ substantially. Second, DC guns are frequently operated with the laser spot shifted from the cathode center that causes a fraction of high energy ions to miss the laser spot. Therefore, a detailed knowledge of the ion spectra and the efficiency of QE damage as a function of the ion energy are required to predict the cathode lifetime with reasonable accuracy.

\section{MITIGATION OF ION BOMBARDMENT}

The presented analysis of the ion motion immediately suggests two mitigation techniques: rf phasing and cathode biasing.

The dependence of the initial drift velocity $\dot{\mathbf{x}}_{0}$ on the ionization phase can be employed to suppress ion bombardment in a single-cell gun. If the force $-e \mathcal{E}(\mathbf{r}) \times$ $\cos (\omega t+\psi)$ acting on electrons is accelerating in the phase range from $-\pi / 2$ to $\pi / 2$, the initial velocity $\dot{\mathbf{x}}_{0}$ points in the direction of acceleration when the phase $\phi_{0}$ is between 0 and $\pi / 2$ and in the opposite direction when $\phi_{0}$ 
is between $-\pi / 2$ and 0 . Thus, the initial drift velocity $\dot{\mathbf{x}}_{0}$ of all ions will point out of the gun if the rf phase changes between 0 and $\pi / 2$ (plus an integer number of full $\mathrm{rf}$ cycles) during the acceleration of electron bunches. Ions with a drift velocity pointing out of the gun and a total effective energy greater than the effective potential energy for all $z$ greater than the ionization coordinate will exit the gun. Only a small portion of ions originating close to the cathode still will be able to strike the cathode. Because a positively charged ion is accelerated towards the cathode immediately after ionization, it can reach the cathode on the first rf cycle after the ionization. If the ion does not strike the cathode on the first rf cycle, it will drift away from the cathode. The distance from which the ion can reach the cathode depends upon the rf phase at which the beam is generated. However, this distance cannot be larger than double the amplitude of the fast oscillations near the cathode. We note that the phase range from 0 to $\pi / 2$ might not be optimal for minimizing the emittance and energy spread.

The phasing method described above is not applicable to a multicell gun because the accelerating phase cannot be limited to the range between 0 and $\pi / 2$. Cathode biasing can be used in this case. As shown in Sec. III A, a bias voltage of a few hundred volts might suffice to significantly reduce the rate of ion bombardment.

\section{CONCLUSIONS}

Using the ponderomotive potential allows for detailed studies of the motion of ions in an rf gun without employing tracking. This method yields the impact energy of ions and the rate of cathode bombardment as functions of the rf frequency, accelerating gradient, and accelerating phase. With this information, mitigation techniques, similar to those we described in the paper, can be developed to reduce the rate of ion backbombardment.

\section{ACKNOWLEDGMENTS}

We would like to thank Ilan Ben-Zvi for bringing the subject of ion bombardment in $\mathrm{rf}$ guns to our attention. This work was performed under the auspices of the U.S. Department of Energy.

\section{APPENDIX A: EFFECT OF ELECTRON BEAM ON ION MOTION IN BNL SRF GUN}

\section{Effect on axial motion}

The interaction between an ion and the field of the electron beam changes the ion momentum and kinetic energy. First, we consider the interaction with a bunch that produces the ion. In general, treating this process for a relativistic beam with image charges is complicated. However, we note that the force on the ion is maximum close to the cathode where the beam is nonrelativistic and the field of the image charges is strongest. For the non- relativistic beam, Eq. (B8) yields the corresponding variation in the ion momentum:

$$
\frac{\delta p}{T_{b}}=\tilde{\mathcal{F}}_{\mathrm{dc}}\left(z_{i}\right),
$$

where $T_{b}$ is the time interval between electron bunches, and $\tilde{\mathcal{F}}_{\mathrm{dc}}\left(z_{i}\right)$ is the electric force produced by a portion of an equivalent $\mathrm{DC}$ beam extending from the ionization coordinate $z_{i}$ to infinity. The equivalent $\mathrm{DC}$ beam has the same parameters as the $\mathrm{CW}$ beam, including the size, velocity profile, and the current, but lacks the time structure. This force can be easily determined by a standard static solver such as Poisson [13].

Equations (6), (8), and (A1) yield the initial effective kinetic energy:

$$
T_{e 0}=\frac{m c^{2}}{2}\left(\frac{\lambda q\left[2 \pi h \mathcal{E}_{b}-\mathcal{E}_{a i} \sin \left(\phi_{0}\right)\right]}{m c^{2}}\right)^{2},
$$

where $h$ is the harmonic number, $\mathcal{E}_{a i}$ is the rf electric field on the gun axis at the ionization point $z_{i}$, and $\mathcal{E}_{b}$ is the electric field at $z_{i}$ produced by the portion of the equivalent DC beam with $z>z_{i}$. Figure 7 shows the initial effective kinetic energy (A2) in the BNL gun for three different bunch intensities: 0 [same as Eq. (8)], $1 \mathrm{nC}$, and $5 \mathrm{nC}$. The beam field was calculated by Poisson. In this simulation, the CW beam was represented by the DC beam with the same average current and the charge density given by

$$
\rho(z)=\frac{I}{v(z) S},
$$

where $v$ is the beam velocity and $S$ is its cross sectional area. This result shows that the effect produced by $1 \mathrm{nC}$ bunches is less than $5 \%$ and is negligible. The variation in the initial effective kinetic energy caused by $5 \mathrm{nC}$ bunches reaches $30 \%$ close to the cathode, where the beam field is maximum. However, even this variation is too small to

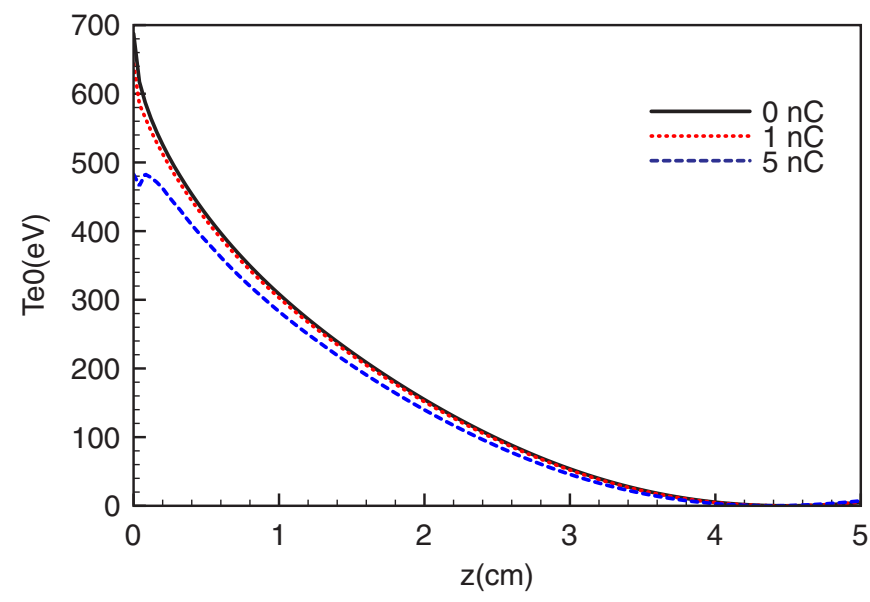

FIG. 7. (Color) Initial effective kinetic energy of ions in the BNL gun for three different beam bunch intensities: 0,1 , and $5 \mathrm{nC}$. 


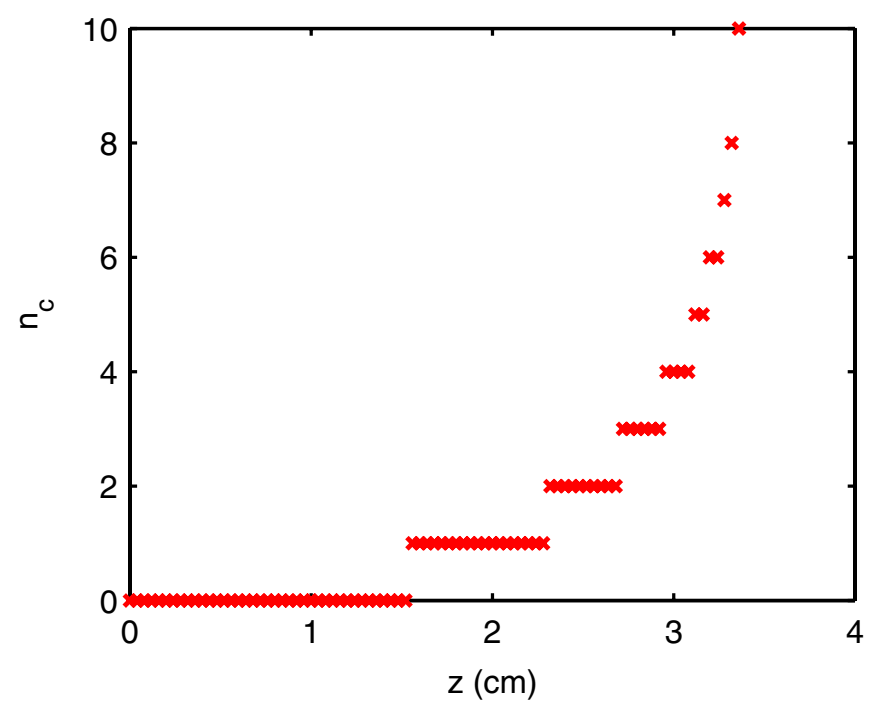

FIG. 8. (Color) Number of ion-bunch collisions as a function of the ionization coordinate. Ions originating beyond the point $z=$ $3.4 \mathrm{~cm}$ do not reach the cathode.

significantly affect the flux of ions bombarding the cathode. We note that the maximum kinetic beam energy for the shown $z$ range is approximately $1 \mathrm{MeV}$. Therefore, the shown change in the effective kinetic energy is overestimated.

After ionization, the ion can interact with more bunches before it reaches the cathode. Figure 8 shows the number of ion-bunch collisions for the $9.38 \mathrm{MHz}$ operational mode $(h=75)$ as a function of the ionization coordinate $z_{i}$. (We omitted the bunch that produces the ion.) Accordingly, if the ion originates between 0 and $1.5 \mathrm{~cm}$, it does not collide with the beam and, thus, is affected only by the bunch that generates the ion. As demonstrated in Appendix B, the total variation of the kinetic energy of the ion in collisions with electron beam bunches can be crudely estimated as a negative variation of the ion potential energy in the equivalent DC beam. Poisson simulations revealed that the potential drop along the axis of the DC beam is approximately equal to $0.2 \mathrm{~V} / \mathrm{mA}$. Noting that the effective kinetic energy of most ions is above $100 \mathrm{eV}$, we can consider only the interaction with bunches that create ions and disregard collisions with other bunches up to a beam current of a few hundred milliamperes, i.e., much higher than the nominal beam current at a $9.38 \mathrm{MHz}$ bunch repetition rate.

The analysis outlined above can significantly overestimate the beam field at the exit of the gun where the beam is relativistic. Instead, the potential difference induced by the variation in the beam size and the transition from the gun cavity to the transfer line can be roughly estimated as [10]

$$
\delta U_{b}=\frac{2 I}{c} \ln \left(\frac{a_{1} b_{2}}{a_{2} b_{1}}\right),
$$

where $a_{1,2}$ and $b_{1,2}$, respectively, are the beam size and the radius of the vacuum pipe at two different locations. Neglecting the variation in the beam size and assuming that the radius of the vacuum pipe is 5 times smaller than the radius of the gun cavity $\left(b_{1} / b_{2} \sim 5\right)$, we obtain $\left|\delta U_{b}\right|=0.08 \mathrm{~V} / \mathrm{mA}$ for the BNL gun. Thus, the potential difference induced by the beam at the exit of the gun can be neglected up to a beam current of a few hundred milliamperes. We note that this number should be approximately the same for all rf guns because of the logarithmic dependence on the pipe radius.

\section{Effect on radial motion}

According to the standard approach described elsewhere (for example, [10]), the transverse motion of ions in the $\mathrm{CW}$ beam is equivalent to the motion in a focusing channel composed of focusing lenses and drifts. Treating such motion involves the standard matrix analysis and is straightforward.

When the beam is the only source of transverse focusing, the motion of ions becomes unstable if the phase advance of ion oscillations per an ion-bunch collision exceeds $\pi$. For a round relativistic beam, the threshold current is given by

$$
I_{\mathrm{th}}=\frac{2 A a^{2} e}{Z r_{p} c} f_{b}^{2}
$$

where $f_{b}$ is the bunch repetition rate, $r_{p}$ is the classical proton radius, and $a$ is the beam radius. Should the ion motion become unstable, the amplitude of ion oscillations will grow exponentially with the number of collisions until it exceeds the beam size. If the beam current is smaller than the threshold current, the ions are confined transversely within the beam.

In principle, ion trajectories can be calculated to almost any degree of accuracy considering individual collisions with electron bunches and solving (14) between the collisions. However, we emphasize that to estimate the rate of cathode ion bombardment it is sufficient to demonstrate which ions can reach the cathode without knowing the exact details of their trajectories. Below the threshold current (A5), the beam focuses ions towards the gun axis and brings more ions to the cathode. Thus, we can assess the rate of cathode ion bombardment without the beam and use this number as a lower boundary estimate of ion bombardment with the beam present. Above the stability threshold, a similar approach can be applied to ions if the amplitude of their oscillations does not grow substantially.

The electron beam with a radius of $2 \mathrm{~mm}$ and a bunch repetition rate of $9.38 \mathrm{MHz}$ will have a net focusing effect on $\mathrm{H}_{2}^{+}$ions in the $\mathrm{BNL}$ gun provided that the current is below $500 \mathrm{~mA}$. As the maximum beam current will not exceed $50 \mathrm{~mA}$ in the $9.38 \mathrm{MHz}$ operational mode, we can safely assume that almost all ions originating between 0 and $3.4 \mathrm{~cm}$ along the gun axis will strike the cathode. 


\section{APPENDIX B: EQUIVALENT DC BEAM}

In this section, we show that the variation of the kinetic energy experienced by an ion in collisions with electron bunches is approximately equal to the variation of the ion potential energy in the equivalent DC beam, which has the same parameters as the $\mathrm{CW}$ beam, including the size, velocity profile, and current, but lacks the time structure. This model includes the space charge field but omits the wakefield induced by the $\mathrm{CW}$ beam.

Treating the interaction between the ion and the beam, we make the following assumptions: (i) we note that the force on the ion is maximum close to the cathode where the beam is nonrelativistic and the field of image charges is strongest. Therefore, to crudely estimate the effect of the beam field on the ion, we can assume that the electron beam is nonrelativistic; (ii) considering that electrons move much faster than ions, we assume that the ion position does not change during a collision with an electron bunch; (iii) we assume that each collision causes only a slight variation of the ion momentum. Under these assumptions, the instantaneous longitudinal force on an ion can be written as an integral of contributions produced by infinitesimally thin charged slices with a radius equal to the local radius of the beam:

$$
\mathcal{F}\left(z_{i}, t\right)=\int_{0}^{\infty} G\left(z, z_{i}\right) d q=\int_{0}^{\infty} G\left(z, z_{i}\right) \lambda(z, t) d z,
$$

where $z_{i}$ is the ion $z$ coordinate, $d q$ is the charge of each slice, and $\lambda(z)=d q / d z$ is the beam linear charge density. The function $G\left(z, z_{i}\right)$ depends on the beam size and includes the field of image charges. This formula is correct for both DC and CW beams. For a DC beam, formula (B1) yields

$$
\mathcal{F}_{\mathrm{dc}}\left(z_{i}\right)=I \int_{0}^{\infty} \frac{G\left(z, z_{i}\right)}{v(z)} d z
$$

where $I$ is the beam current.

Considering the interaction of the ion with the $\mathrm{CW}$ beam, first, we analyze collisions with bunches that did not generate the ion. To simplify the analysis, we assume that the bunch distribution is given by the delta function:

$$
\lambda(z, t)=Q_{b} \delta\left(z-\int_{0}^{t} v(t) d t\right),
$$

where $Q_{b}$ is the bunch charge. The full variation of the ion momentum in a collision is given by

$$
\begin{aligned}
\delta p & =\int_{0}^{\infty} \mathcal{F}\left(z_{i}, t\right) d t \\
& =Q_{b} \int_{0}^{\infty} \int_{0}^{\infty} G\left(z, z_{i}\right) \delta\left(z-\int_{0}^{t} v d t\right) d z d t .
\end{aligned}
$$

Integrating over $t$ and dividing the result by the time interval between bunches, we obtain

$$
\frac{\delta p}{T_{b}}=I \int_{0}^{\infty} \frac{G\left(z, z_{i}\right)}{v(z)} d z=\mathcal{F}_{\mathrm{dc}}\left(z_{i}\right) .
$$

Thus, the variation of the ion momentum in a collision with a bunch, divided by the time interval between bunches, is equal to the force produced by the equivalent DC beam.

The total variation of the ion energy is a sum over collisions experienced by the ion:

$$
\begin{aligned}
\delta T & =\sum \delta T_{j} \approx \sum \frac{p_{j} \delta p_{j}}{m}=\sum v_{j} \delta p_{j}=\sum v_{j} T_{b} \frac{\delta p_{j}}{T_{b}} \\
& =\sum \delta z_{j} \mathcal{F}_{\mathrm{dc}, j},
\end{aligned}
$$

where the subscript $j$ denotes individual collisions and $\delta z_{j}$ is the product $v_{j} T_{b}$ approximately equal to the distance the ion travels between collisions. The sum $\sum \delta z_{j} \mathcal{F}_{\mathrm{dc}, j}$ is a piecewise approximation of the integral $\int \mathcal{F}_{\mathrm{dc}}(z) d z$. Thus, the total variation of the ion kinetic energy is approximately equal to the negative variation of its potential energy in the equivalent DC beam.

A similar analysis can be applied to the interaction with the bunch generating the ion. However, in this case, the lower limit of the time integral has to be changed from 0 to the time it takes for the bunch to reach the ionization coordinate, $z_{i}$. This yields the momentum variation:

$$
\frac{\delta p}{T_{b}}=I \int_{z_{i}}^{\infty} \frac{G\left(z, z_{i}\right)}{v(z)} d z=\tilde{\mathcal{F}}_{\mathrm{dc}}\left(z_{i}\right) .
$$

Thus, the variation of the ion momentum caused by the bunch producing the ion, divided by the time interval between the bunches, is equal to the force produced by the portion of the equivalent DC beam extending from $z_{i}$ to infinity. We note that the momentum variation (B8) is proportional to the bunch charge.

\section{APPENDIX C: MOTION OF IONS OFF AXIS, FIRST ITERATION}

Equation (14) can be solved iteratively. If the trajectory radius changes little, we can limit the solution to the first iteration. That is, we write the ion trajectory radius as $r=$ $r_{0}+r_{1}$, where $r_{0}$ is the initial radius, and the equation for the first iteration $r_{1}$ as

$$
m \ddot{r}_{1}=F_{r}\left(r_{0}\right) .
$$

The standard method of variation of constants yields the solution of $(\mathrm{C} 1)$

$$
r_{1}(t)=\int_{0}^{t} \frac{F_{r}}{m}(t-\tau) d \tau+\dot{x}_{r 0} t
$$

where $\dot{x}_{r 0}$ is the $r$ projection of $\dot{\mathbf{x}}_{0}$. It is convenient to change the independent variable from $t$ to $z$ according to

$$
t=\int_{z_{i}}^{z} \frac{d \xi}{\dot{x}_{z}(\xi)},
$$


where $\dot{x}_{z}$ is the $z$ projection of $\dot{\mathbf{x}}$ and $z_{i}$ denotes the ionization coordinate. We note that $\dot{x}_{z}$ depends on $z_{i}$ as a parameter. Thus, $r_{1}(z)$ can be written as

$$
r_{1}(z)=\int_{z}^{z_{i}} \frac{F_{r}\left(\xi^{\prime}\right) d \xi^{\prime}}{m \dot{x}_{z}\left(\xi^{\prime}\right)} \int_{z}^{\xi^{\prime}} \frac{d \xi^{\prime \prime}}{\dot{x}_{z}\left(\xi^{\prime \prime}\right)}-\left(\frac{d r}{d z}\right)_{0} \int_{z}^{z_{i}} \frac{\dot{x}_{z 0} d \xi}{\dot{x}_{z}(\xi)} .
$$

Using Eqs. (5), (7), (8), and (C4) and taking into account $\dot{x}_{r} \ll \dot{x}_{z}$, we obtain the radial deviation of the ion trajectory at the cathode, $r_{1 c}=r_{1}(0)$, normalized to $r_{0}$ :

$$
\begin{aligned}
\frac{r_{1 c}}{r_{0}}= & \int_{0}^{z_{i}} \frac{\left[\mathcal{E}_{a}\left(\mathcal{E}_{a}^{\prime \prime}+\frac{\mathcal{E}_{a}}{\hbar^{2}}\right)-\frac{\mathcal{E}_{a}^{\prime 2}}{2}\right] d \xi^{\prime}}{2 \sqrt{\mathcal{E}_{a i}^{2}\left\{1+2 \sin ^{2}\left[\phi\left(z_{i}\right)\right]\right\}-\mathcal{E}_{a}^{2}}} \\
& \times \int_{0}^{\xi^{\prime}} \frac{d \xi^{\prime \prime}}{\sqrt{\mathcal{E}_{a i}^{2}\left\{1+2 \sin ^{2}\left[\phi\left(z_{i}\right)\right]\right\}-\mathcal{E}_{a}^{2}\left(\xi^{\prime \prime}\right)}} \\
& +\frac{\mathcal{E}_{a i}^{\prime}}{2 \mathcal{E}_{a i}} \int_{0}^{z_{i}} \sqrt{\frac{2 \mathcal{E}_{a i}^{2} \sin ^{2}\left[\phi\left(z_{i}\right)\right]}{\mathcal{E}_{a i}^{2}\left\{1+2 \sin ^{2}\left[\phi\left(z_{i}\right)\right]\right\}-\mathcal{E}_{a}^{2}}} d \xi,
\end{aligned}
$$

where $\mathcal{E}_{a i}$ denotes $\mathcal{E}_{a}\left(z_{i}\right)$. Equation (C5) is valid only if the inequality $r_{1}(z) / r_{0} \ll 1$ is satisfied for all $z$.

\section{APPENDIX D: EFFECT OF MAGNETIC FIELD}

The rf guns, superconducting and normal conducting, typically are equipped with one or more solenoids to preserve the transverse emittance of the beam. In a normal-conducting rf gun, an emittance compensating solenoid usually is sited at the exit port of the gun cavity. Also, a normal-conducting gun can be equipped with a bucking solenoid to cancel out the solenoidal field at the cathode. The solenoids are aligned coaxially with the rf cavity. Because both the rf field and the solenoidal magnetic field are azimuthally symmetric, the canonical angular momentum is constant:

$$
P_{\varphi}=m r^{2} \dot{\varphi}+\frac{q A_{\varphi} r}{c}=\text { const. }
$$

Equation (D1) along with the initial condition $\dot{\varphi}_{0}=0$ describes the angular ion motion. The averaged $r z$ motion is described by the Hamiltonian function

$$
H=\frac{p_{r}^{2}}{2 m}+\frac{p_{z}^{2}}{2 m}+\Psi
$$

with the effective potential energy [19]:

$$
\Psi=\frac{\left(P_{\varphi}-q A_{\varphi} r / c\right)^{2}}{2 m r^{2}}+U_{e}
$$

where $U_{e}$ is the effective rf potential energy. If the radius of an ion trajectory is small compared to the inner radius of the solenoid, the potential $A_{\varphi}(z)$ is approximately equal to $B(z) r / 2$, where $B(z)$ is the magnetic field on the axis.

The Hamiltonian or Lagrange equations with the potential given by (D3) can be solved numerically to accurately calculate ion trajectories. However, in many cases, a crude estimate of the effect suffices. This can be easily obtained if the hard-edge approximation is applicable:

$$
\frac{1}{2 \pi} \frac{q B_{m} L_{e}}{\left(2 m c^{2} T_{e}\right)^{1 / 2}} \ll 1,
$$

where $B_{m}$ is the maximum field, $L_{e}$ is the characteristic length of the field edge, and $T_{e}$ is the effective kinetic energy of an ion. Equation (D4) also can be rewritten in practical units:

$$
8 \times 10^{-4} \frac{B_{m}(\mathrm{G}) L_{e}(\mathrm{~cm})}{\left[A T_{e}(\mathrm{eV})\right]^{1 / 2}} \ll 1,
$$

where $B_{m}$ is expressed in Gauss, $L_{e}$ in centimeters, and $T_{e}$ in electron volts. If inequality (D4) is satisfied, the radius of an ion trajectory changes little as an ion crosses the field edge. The adiabatic condition, which is opposite to (D4), is not satisfied for a typical range of parameters.

As an ion crosses the field edge, it gains angular velocity $\dot{x}_{\varphi}$. Accompanying the appearance of the angular velocity is a reduction in the axial velocity. If the hard-edge approximation is applicable, the reduction of the axial velocity is given by

$$
\frac{\delta \dot{x}_{z}}{\dot{x}_{z}} \approx-\frac{\dot{x}_{\varphi}^{2}}{2 \dot{x}_{z}^{2}}=-\frac{q^{2} B_{m}^{2} r^{2}}{16 m c^{2} T_{e}} .
$$

Comparing (D6) to (D4) and considering that the beam size typically is much smaller than the length of the solenoidal field edge, one can conclude that the effect of the magnetic field on the longitudinal motion of ions is negligible if (D4) is satisfied.

The radial motion strongly depends on where ionization occurs. If an ion originates in a field free region and enters the solenoidal field, the effective potential energy (D3) is given by

$$
\Psi=\frac{q^{2} B^{2}(z)}{8 m c^{2}} r^{2} .
$$

This focusing potential will keep the radial motion of ions bound.

Should an ion ionized inside the solenoid leave the field, the effective potential energy (D3) in the field free region becomes

$$
\Psi=\frac{q^{2} B_{0}^{2} r_{0}^{4}}{8 m c^{2} r^{2}},
$$

where $r_{0}$ is the radius of the ionization point and $B_{0}$ is the magnetic field on the axis at $z$ equal to that of the ionization point. The defocusing effective potential (D8) is caused by the angular velocity gained by the ion as it leaves the field. If the initial radial velocity and other potential contributions are zero, the radius of the ion trajectory in the potential energy (D8) is 


$$
r(t)=r_{0} \sqrt{1+\frac{\dot{x}_{\varphi}^{2}}{r_{0}^{2}} t^{2}},
$$

where $t$ is time after the ion left the field. The growth of the trajectory radius can engender a reduction of the ion flux per unit area. If the axial velocity $\dot{x}_{z}$ does not change significantly over a distance $d$, we can substitute $d / \dot{x}_{z}$ for $t$ in the last equation. If $d$ is not significantly larger than $L_{e}$ and (D4) is satisfied, the growth of the trajectory radius is small.

Thus, we can conclude that the effect of the solenoidal magnetic field can be neglected provided that the hardedge condition (D4) is satisfied. When this condition is not met, ion trajectories can be calculated numerically.

[1] V. Litvinenko et al., in Proceedings of the 21st Particle Accelerator Conference, Knoxville, 2005 (IEEE, Piscataway, NJ, 2005), p. 2768.

[2] J. Kewisch, I. Ben-Zvi, T. Rao, A. Burrill, D. Pate, R. Grover, R. Todd, H. Bluem, D. Holmes, and T. Schultheiss, in Proceedings of the Workshop on Polarized Sources, Targets and Polarimetry-PSTP 2007, BNL, Upton, New York, 2007 (AIP, Melville, New York, 2007), p. 118.

[3] A. V. Aleksandrov, N. S. Dikansky, R. G. Gromov, and P. V. Logatchov, in Proceedings of the 18th Particle Accelerator Conference, New York, 1999 (IEEE, Piscataway, New Jersey, 1999), p. 78.

[4] R. Fliller, T. Anderson, H. Edwards, H. Bluem, T. Schultheiss, C. Sinclair, and M. Huening, in Proceedings of the 21st Particle Accelerator Conference, Knoxville, 2005 (Ref. [1]), p. 2708.
[5] J. W. Lewellen, Phys. Rev. ST Accel. Beams 5, 020101 (2002).

[6] P. L. Kapitza, Zh. Eksp. Teor. Fiz. 21, 588 (1951).

[7] L. D. Landau and E. M. Lifshitz, Mechanics (Course of Theoretical Physics) (Butterworth-Heinemann, Burlington, MA, 1982), 3rd ed.

[8] H. A. H. Boot and R. B.R.-S. Harvie, Nature (London) 180, 1187 (1957).

[9] A. V. Gaponov and M. A. Miller, Zh. Eksp. Teor. Fiz. 34, 242 (1958) [Sov. Phys. JETP 7, 168 (1958)].

[10] A. Poncet, in Proceedings of CERN Accelerator School: 5th Advanced Accelerator Physics Course, Rhodes, Greece, 1993 (CERN, Geneva, 1995), p. 859.

[11] R. Calaga, I. Ben-Zvi, M. Blaskiewicz, X. Chang, D. Kayran, and V. Litvinenko, Physica (Amsterdam) 441C, 159 (2006).

[12] V. Litvinenko et al., in Proceedings of EPAC08, Genoa, Italy, 2008 (EPS-AG/CERN, Geneva, 2008), p. 193, http:// accelconf.web.cern.ch/AccelConf/e08/papers/ mopc057.pdf.

[13] K. Halbach and R. Holsinger, Part. Accel. 7, 213 (1976).

[14] L. Young, LANL Technical Report No. LA-UR-96-1835, 1996.

[15] MATHCAD, PTC Corporate Headquarters, 140 Kendrick Street, Needham, MA 02494, USA (2008), http:// www.ptc.com/products/mathcad/.

[16] W. H. Press, S. A. Teukolsky, W. T. Vetterling, and B.P. Flannery, Numerical Recipes in $C$ (2nd ed.): The Art of Scientific Computing (Cambridge University Press, New York, 1992), ISBN 0-521-43108-5.

[17] M. Reiser, Theory and Design of Charged Particle Beams (John Wiley \& Sons, Inc., New York, 1994).

[18] E. Pozdeyev, Phys. Rev. ST Accel. Beams 10, 083501 (2007).

[19] G. Schmidt, Physics of High Temperature Plasmas (Academic Press, New York, 1966). 